Pacific Journal of Mathematics

RIGHT SUBDIRECTLY IRREDUCIBLE SEMIGROUPS

October 1979 


\title{
RIGHT SUBDIRECTLY IRREDUCIBLE SEMIGROUPS
}

\author{
S. A. Rankin, C. M. Reis and G. Thierrin
}

It is well-known that a semigroup is subdirectly irreducible if and only if it has a minimum nontrivial congruence. From this point of view, it is natural to call a semigroup right (left) subdirectly irreducible if and only if it has a minimum nontrivial right (left) congruence. It turns out that such semigroups are exactly the subdirectly irreducible semigroups for which the minimum nontrivial congruence is also a minimum nontrivial right (left) congruence. These semigroups form a class of subdirectly irreducible semigroups for which results similar to those obtained by Schein for commutative subdirectly irreducible semigroups are obtained. In fact, since a commutative semigroup is subdirectly irreducible if and only if it is right subdirectly irreducible, some of the results of this paper offer additional knowledge on the structure of subdirectly irreducible semigroups of the third kind.

The set of all right subdirectly irreducible semigroups will be partitioned, for the purpose of investigation, into ten classes, each class being defined in terms of idempotents. Six of these classes contain exactly one semigroup each. Several of these semigroups have also been described in a related study by Baird and Thierrin [1]. A right subdirectly irreducible semigroup $S$ does not belong to any of these six exceptional classes if and only if the set of idempotents $E(S)$ of $S$ is contained in $\{0,1\}$. The remaining four classes of right subdirectly irreducible semigroups correspond then to the four possible subsets of $\{0,1\}$.

As for notation, we shall let $\mathscr{N}$ denote the set of natural numbers. If $S$ is a semigroup and $a \in S$, we shall let $\lambda_{a}: S \rightarrow S$ denote left translation of $S$ by a (i.e., $x \rightarrow a x$ for all $x \in S$ ) and $\langle a\rangle=\left\{a^{i} \mid i \in \mathscr{N}\right\}$. If $H \subset S,|H|$ shall denote the cardinality of $H$. Moreover, we shall define a right congruence $\phi_{H}$ on $S$ by $x \equiv y\left[\phi_{H}\right]$ if and only if $H x=H y$. If $H$ is a singleton, say $H=\{a\}$, then we denote $\phi_{H}$ by $\phi_{a}$. Finally, if $\phi$ is any equivalence relation on $S$, let $\dot{\phi}(a)$ denote the equivalence class of $a \in S$.

2. Right subdirectly irreducible semigroups. It is clear that a right subdirectly irreducible semigroup must have a minimum nontrivial (i.e., not a singleton) right ideal. Since every left translate of a minimal right ideal is a minimal right ideal, the minimum right ideal of a right subdirectly irreducible semigroup is a two- 
sided ideal, called the core [5] of $S$, and denoted by $K=K(S)$. The minimum right congruence shall be denoted throughout by $\rho=\rho(S)$. All nontrivial $\rho$-classes are contained in $K$.

LEMMA 2.1. Let $S$ be right subdirectly irreducible and $a \in S$. If there exists $x \in S$ such that $|a \rho(x)|>1$, then $\lambda_{a}$ is injective.

Proof. If $\lambda_{a}$ is not injective then $\phi_{a} \neq \varepsilon$ and so $\rho \leqq \phi_{a}$.

A right subdirectly irreducible semigroup is obviously subdirectly irreducible. With the preceding lemma, we can say more.

THEOREM 2.2. The minimum nontrivial right congruence on a right subdirectly irreducible semigroup is a two-sided congruence.

Proof. Let $S$ be right subdirectly irreducible. If $a \in S$ is such that $\lambda_{a}$ is not injective, then $|a \rho(x)|=1$ for all $x \in S$. On the other hand, if $\lambda_{a}$ is injective, then $|a K|>1$ and so $a K=K$. Define a congruence $\rho_{a}$ by $x \equiv y\left[\rho_{a}\right]$ if $a x \equiv a y[\rho]$. Let $x$ and $y$ be distinct elements of $K$ such that $x \rho y$. Then $x=a s$ and $y=a t$ for distinct $s$ and $t$ from $S$. But then $s \equiv t\left[\rho_{a}\right]$ and so $\rho_{a} \neq \varepsilon$. Thus $\rho \leqq \rho_{a}$ and so $x \rho y$ implies axpay.

We proceed now to investigate the set $E(S)$ of idempotents of a right subdirectly irreducible semigroup $S$. It will be shown that except for six exceptional semigroups, the set of right subdirectly irreducible semigroups can be partitioned for investigation according to the following four types:

(i) $E(S)=\{1\}$

(ii) $E(S)=\{1,0\}$

(iii) $E(S)=\{0\}$

(iv) $E(S)=\varnothing$.

Of these four cases, the type (ii) are the most accessible. We have been able to say very little about the remaining types (i), (iii) and (iv).

THEOREM 2.3. Each idempotent of a right subdirectly irreducible semigroup is either a left zero or a left identity.

Proof. Suppose $e \in E(S)$ is not a left zero. Then $e S \neq e$ and so $K \subset e S$. But then $\phi_{e}$ restricted to $K$ is the identity and so $\rho$ does not refine $\phi_{e}$. Thus $\phi_{e}=\varepsilon$ and since for all $a \in S, e a \equiv a\left[\phi_{e}\right], e a=a$ and so $e$ is a left identity for $S$.

LEMMA 2.4. Let $S$ be right subdirectly irreducible with a left identity $e$ which is not a right identity. Then $\rho$ has exactly one 
nontrivial class $\rho(a)$, where $a \notin S e$ and $S=S e \cup\{a\}, \rho(a)=\{a, a e\}$.

Proof. Since $e$ is not a right identity, there exists $a \in S$ such that $a e \neq a$. Define a right congruence $\phi$ on $S$ by $x \equiv y$ iff $x, y \in$ $\{a, a e\}$ or $x=y$. Since this right congruence is not $\varepsilon$, it must be $\rho$. But then for the left identity $e$, there is at most one such element $a$ and so for all $x \in S \backslash\{a\}, x e=x$. Thus $S=S e \cup\{a\}, a \notin S e$.

CoROLlaRY 2.5. If $S$ is right subdirectly irreducible and has exactly one left identity (not a right identity), then $S$ has a left zero.

Proof. If $e$ is the left identity of $S$, then $S=S e \cup\{a\}, a \notin S e$ and $\rho(a)=\{a, a e\}$. If $a^{2}=a$, then $a$ is $a$ left zero and $a e=a$, a contradiction. Thus $a^{2} \in S e$. If $a \in a S$, say $a=a x$, then $x \neq a$. Thus $a x e=a x=a$ whence $a e=a$, again a contradiction and so $a \notin a S$. Since $\rho(a)=\{a, a e\} \subset K, \rho(a)$ must be contained in every nontrivial right ideal and so $|a S|=1$. Thus $a S=\{a e\}$ and $S=e S$ whence $a e S=\{a e\}$.

THEOREM 2.6. A right subdirectly irreducible semigroup has at most two left identities. A semigroup $S$ is right subdirectly irreducible with two left identities iff $S$ is the right zero semigroup of order 2 with or without an adjoined zero.

Proof. It is easily seen that the right zero semigroup of order 2 with or without an adjoined zero is right subdirectly irreducible with $K=S$. If $S$ has no zero, then $\rho=\omega$, the universal congruence. Otherwise $\rho$ is the principal congruence [5] of the zero.

Now let $S$ be right subdirectly irreducible with a left identity $e$. Then $S=S e \cup\{a\}, a \notin S e$. If $f$ is a left identity for $S$, then either $f=a$ or $f \in S e$ whence $e=f e=f$. Thus if $e \neq f$ we have $f=a$ and $S=S e \cup\{f\}$. Similarly, $S=S f \cup\{e\}, e \notin S f$. Thus $S=$ $(S e \cap S f) \cup\{e, f\}$ and $I=S e \cap S f$ is an ideal. If $|I|>1$ then the Rees congruence for $I$ is not refined by $\rho$ since $\rho(e)=\rho(f)=\{e, f\}$. Thus $|I| \leqq 1$ and so $I=\varnothing$ or else $S$ has a zero and $I=\{0\}$.

THEOREM 2.7. A semigroup $S$ is right subdirectly irreducible with a unique left identity $e$ (which is not a right identity) iff $S=\{a, e, 0\}$ with $a^{2}=a e=0$.

Proof. Clearly $S=\{a, e, 0\}$ is right subdirectly irreducible with $K=\{a, 0\}$ and $\rho$ the Rees congruence of $K$. On the other hand, if $S$ is right subdirectly irreducible with a unique left identity $e$ which 
is not a right identity, then $S=S e \cup\{a\}, a \notin S e$; and $f=a e=a^{2}$ is a left zero, so $K=\{a, f\}$. For each $x \in S$, either $x a=a$ or else $x a=f$ and occordingly, either $x f=x a e=a e=f$ or else $x f=x a e=$ $f e=f$. Thus $f=0$. Define $S_{1}=\{x \in S \mid x a=a\}$ and $S_{2}=\{x \in S \mid x a=0\}$. Thus $S_{1}$ and $S_{2}$ form a partition of $S$ and $S_{2}$ is an ideal. Since $a \in S_{2},\left\{S_{1}, S_{2} \mid\{a\},\{a\}\right\}$ defines a right congruence on $S$ which is not refined by $\rho$, whence $\left|S_{1}\right| \leqq 1,\left|S_{2}\right|\{a\} \mid \leqq 1$ and so $S_{1}=\{e\}, S_{2}=\{a, 0\}$.

LEMMA 2.8. A right subdirectly irreducible semigroup has at most two left zeroes. If there is exactly one left zero, it is a zero, while if there are two left zeroes, $e$ and $f$, then $K=\{e, f\}$.

Proof. Let $e$ be a left zero of a right subdirectly irreducible semigroup $S$. Then for all $a \in S$, ae is a left zero. If $S$ has only one left zero, $a e=e$ for all $a \in S$ whence $e$ is a zero. If $S$ has more than one left zero, then since each subset of the set of all left zeroes is a right ideal, $S$ has exactly two left zeroes and they form the minimum right ideal.

THEOREM 2.9. A semigroup $S$ is right subdirectly irreducible with two left zeroes iff $K=\left\{e_{1}, e_{2}\right\}$ is the left zero semigroup of order 2 and $S$ is one of the following semigroups:

$$
\begin{array}{ll}
\text { (i) } & S=K \\
\text { (ii) } & S=K^{1} \\
\text { (iii) } & S=K^{1} \cup\{a\}, a^{2}=1, \\
& a e_{1}=e_{2}, a e_{2}=e_{1} .
\end{array}
$$

Proof. If $S=K$ or $K^{1}$, then $S$ is obviously right subdirectly irreducible with the Rees congruence of $K$ as the minimum right congruence. If $S=K^{1} \cup\{a\}$, then $\{a, 1\}$ is a group and $K$ an ideal and again the Rees congruence of $K$ is a minimum right congruence.

Conversely, if $S$ is right subdirectly irreducible with two left zeroes $e_{1}$ and $e_{2}$, then $K=\left\{e_{1}, e_{2}\right\}$ and we consider the right ideals $\left\{x \mid x K=e_{1}\right\}$ and $\left\{x \mid x K=e_{2}\right\}$. Since they are disjoint and both meet $K$, they must each be singletons. Thus for $a \in S \backslash K, \lambda_{a}$ determines a permutation of $K$. Let $S_{1}=\left\{x \in S \backslash K \mid x e_{1}=e_{1}\right\}$ and $S_{2}=\{x \in$ $\left.S \backslash K \mid x e_{1}=e_{2}\right\}$. Then $\left\{S_{1}, S_{2},\left\{e_{1}\right\},\left\{e_{2}\right\}\right\}$ is a partition of $S$ which defines a right congruence. This congruence is not refined by $\rho$ and so $\left|S_{1}\right| \leqq 1,\left|S_{2}\right| \leqq 1$. If $S_{1} \neq \varnothing$, then $S_{1}=\{e\}$ and $e^{2}=e \notin\left\{e_{1}, e_{2}\right\}$, whence by Theorem $2.3 e$ is a left identity for $S$. Since $S_{2} S_{1} \subset S_{2}$ and $K e=K, e$ is also a right identity for $S$ and so $e=1$. Thus if $S_{2}=\varnothing$ then $S=K^{1}$ while if $S_{2}=\{a\}$ then $a^{2}=1$ since $S_{2} S_{2} \subset S_{1}$.

3. Right subdirectly irreducible monoids. From now on, we 
consider only right subdirectly irreducible semigroups $S$ for which $E(S) \subset\{0,1\}$. If $S$ is a semigroup for which $E(S) \subset\{0,1\}$, then $S$ is right subdirectly irreducible iff $S^{1}$ is right subdirectly irreducible. Thus we need only consider right subdirectly irreducible monoids.

THeOREM 3.1. Let $M$ be a right subdirectly irreducible monoid. Then $M$ is a group or else there exists a subgroup $G=G(M)$ of $M$ whose identity is 1 and $I=M \backslash G$ is an ideal of $M$.

Proof. Suppose $a x=1$ for $a, x \in M$. Then $x a x a=x a$ and so $x a \in E(M)$. If $x a=0$ then $a=a x a=0$ which is not possible. Thus $x a=1$. That is to say, every left divisor of 1 is a right divisor of 1 and the result follows from Lemma 2.9 of [4].

Leмma 3.2. Let $S$ be a right subdirectly irreducible semigroup. Then for $x, y \in S, x y=y$ iff $x=1$ or $y=0$.

Proof. Let $y \in S, y \neq 0$, whence $K \subset y S$. If there exists $x \in S$ such that $x y=y$, then for $k \in K$ we have $k=y t$ for some $t \in S$. But then $x k=k$ and so $x$ is a left identity on $K$. The right congruence defined by $a \equiv b$ if $\langle x\rangle a \cap\langle x\rangle b \neq \varnothing$ is then the identity on $K$, hence the identity on $S$. But $x a \equiv a$ for all $a \in S$ and so $x a=a$ for all $a \in S$. Thus $x$ is a left identity for $S$ and so $x=1$.

CoRollary 3.3. Let $S$ be right subdirectly irreducible. If $S$ is not a group then for all $a \in K, K a \neq K$.

Proof. If $K a=K$ then $t a=a$ for some $t \in K$. Since $a \neq 0$, $t=1$. But if $1 \in S$ and $S$ is not a group, then $S=G \cup I$ with $1 \in G$, $K \subset I$ and $G \cap I=\varnothing$. Thus $1 \notin K$, a contradiction and so $K a \neq K$.

CoRollary 3.4. If $M$ is a right subdirectly irreducible monoid then for all nonzero $a \in M,|G a|=|G|$.

Proof. If $g a=h a$ for $g, h \in G$, then $g^{-1} h=1$ whence $g=h$.

Note that a group is right subdirectly irreducible iff it has a minimum nontrivial subgroup.

THEOREM 3.5. Let $M$ be a right subdirectly irreducible monoid. Then $G$ is right subdirectly irreducible or $G=\{1\}$.

Proof. Suppose $|G|>1$ and let $\left\{G_{\alpha} \mid \alpha \in \Lambda\right\}$ be the set of all 
nontrivial subgroups of $G$. Let $H=\cap\left\{G_{\alpha} \mid \alpha \in \Lambda\right\}$. Denote the right congruence $\phi_{G_{\alpha}}$ by $\phi_{\alpha}$. Then $\rho \leqq \phi_{\alpha}$ for all $\alpha \in \Lambda$. Let $a \in M$ be such that $|\rho(a)|>1$, say $b \in \rho(a), b \neq a$. Since $\rho(a) \subset \phi_{\alpha}(a)=G_{\alpha} a$, then for each $\alpha \in \Lambda$ there exists $g_{\alpha} \in G_{\alpha}, g_{\alpha} \neq 1$, such that $b=g_{\alpha} a$. Thus $g_{\alpha} a=g_{\beta} a$ for all $\alpha, \beta \in \Lambda$ and so $g_{\alpha}^{-1} g_{\beta} a=a$. If $a=0$ then $\rho(a) \subset$ $G_{\alpha} a=0$ implies that $b=0=a$, a contradiction. Thus $a \neq 0$ and so $g_{\alpha}=g_{\beta}$ i.e., $g_{\alpha} \in G_{\beta}$ for all $\beta \in \Lambda$ whence $|H|>1$.

We shall use $H$ to denote the minimum subgroup of a right subdirectly irreducible group $G$.

From McAlister and O'Carroll [3] it is known that a right subdirectly irreducible group is a $p$-group for some prime $p$ and $|H|=p$.

THEOREM 3.6. Let $G$ be a right subdirectly irreducible group. Then $H$ is contained in the center of $G$.

Proof. Let $b \in G, a \in H$. Then since $H$ is normal in $G, b a b^{-1}=a^{j}$ for some integer $j$, and so for each $m \in \mathscr{N}, b^{m} a b^{-m}=a^{j^{m}}$. If $|\langle b\rangle|=p^{n}$, then we have $a=b^{p^{n}} a b^{-p^{n}}=a^{t}$ for $t=j^{p^{n}}$, whence $t \equiv 1(\bmod p)$. But $t \equiv j(\bmod p)$ and so $j \equiv 1(\bmod p)$. Thus $b a b^{-1}=a$.

So we have seen that if $M$ is a right subdirectly irreducible monoid, then $M$ is the disjoint union of a group $G$ and an ideal $I$ (or $I=\varnothing$ ), where if $|G|>1$, then $G$ is a $p$-group with minimum subgroup $H,|H|=p$.

THeOREM 3.7. Let $M$ be a right subdirectly irreducible monoid with $|G|>1$. Then $G$ is a p-group and each nontrivial o-class contains $p$ elements. In fact, if $|\rho(a)|>1$ then $\rho(a)=H a$.

Proof. Since $\rho \leqq \phi_{H}$ we have $\rho(a) \subset H a$. Thus for $b \in \rho(a), b \neq a$, we have $b=h^{i} a$ for some $1 \leqq i \leqq p-1$ and so $a \rho h^{i} a$ implies $h^{i} a \rho h^{2 i} a$. By induction we obtain $\left\{a, h^{i} a, h^{2 i} a, \cdots, h^{(p-1) i} a\right\} \subset \rho(a)$. Since $h^{k i} a \neq a$ for $1 \leqq k \leqq p-1, \rho(a)=H a$.

CoRollary 3.8. Let $M$ be a right subdirectly irreducible monoid with $|G|>1$. If $M$ has a zero then $|\rho(0)|=1$, i.e., 0 is not disjunctive.

Proof. If $|\rho(0)|>1$, then $\rho(0)=H \cdot 0=0$, a contradiction. Thus if $|G|>1,0$ cannot be disjunctive.

CoROLlaRy 3.9. Let $M$ be a right subdirectly irreducible monoid 
with $|G|>1$. Then $K$ is the union of nontrivial $\rho$-classes (union zero if $M$ has a zero).

Proof. If for some $a \in K, x \in M$ we have $|\rho(a)|>1$ but $|\rho(a x)|=1$ then for each $h \in H$, a $\rho h a$ and so $a x=h a x$. Since $H \neq 1$, this implies that $a x=0$ or else no such $x \in M$ exists. Thus the union of the nonsingleton $\rho$-classes (union zero if $M$ has a zero) is a right ideal.

THEOREM 3.10. Let $M$ be a right subdirectly irreducible monoid with zero. If $a I=0$ for some nonzero $a \in K$, then $K=a G \cup 0$. Furthermore, if $|G|>1$, then $K \backslash 0$ is the union of nontrivial o-classes.

Proof. $\quad(a G \cup 0) M=(a G \cup 0)(G \cup I)=a G \cup a G I=a G \cup 0$. Thus $K=a G \cup 0$. Since $G$ acts transitively on the right of $a G$, each $o$-class in $K \backslash 0$ is nontrivial.

Lemma 3.11. Let $M$ be a right subdirectly irreducible monoid. Then for all $a \in M / K, a I \neq 0$.

Proof. If $a \in G$ then $a I=I$. Suppose now that for some $a \in I \backslash K$, $a I=0$. Then $\{x \in I \backslash K \mid x I=0\} \cup 0$ is a nontrivial right ideal which does not contain $K$, a contradiction.

4. Periodic right subdirectly irreducible semigroups.

THEOREM 4.1. Let $S$ be right subdirectly irreducible. If $a \in S$ is aperiodic, then $\lambda_{a}$ is not injective.

Proof: Since $a$ is aperiodic, the right congruence $x \equiv y$ if $\langle a\rangle x \cap\langle a\rangle y \neq \varnothing$ is nontrivial and so $x \rho y$ implies $\langle a\rangle x \cap\langle a\rangle y \neq \varnothing$. Suppose then that $x \rho y$ but $x \neq y$. Now $0 \in\langle a\rangle x$ iff $0 \in\langle a\rangle y$ whence there is a smallest $n \in \mathscr{N}$ such that $a^{n} x=0$. Thus $t=a^{n-1} x \neq 0$ and $a t=0=a 0$ whence $\lambda_{a}$ is not injective. Suppose now that $0 \notin$ $\langle a\rangle x \cup\langle a\rangle y$. We have $a^{k} x=a^{j} y$ for some $j, k \in \mathscr{N}$. If $a^{p} x=a^{q} y$ for some $p, q \in \mathscr{N}$, with $p \geqq k$, then $a^{q} y=a^{p-k} a^{k} x=a^{p-k} a^{j} y=$ $a^{p-k+j} y$. If $q>p-k+j$, say $q=m+p-k+j$, then $a^{m}\left(a^{p-k+j} y\right)=$ $a^{p-k+j} y$ whence $a^{m}=1$, a contradiction. We obtain a similar contradiction if $q<p-k+j$. Thus $p-q=k-j$. Let $n_{0}=k-j$. Then if $a^{r} x=a^{s} y, r-s=n_{0}$. Now for any $t \in \mathscr{N},\left\langle a^{t}\right\rangle x \cap\left\langle a^{t}\right\rangle y \neq \varnothing$ and so for some $r, s \in \mathscr{N}, a^{r t} x=a^{s t} y$ whence $(r-s) t=n_{0}$. Thus each $t \in \mathscr{N}$ divides $n_{0}$ and so $n_{0}=0$. We then have $a^{k} x=a^{k} y$ for some $k \in \mathscr{N}$ and we may assume that $k$ is the least such natural number. Thus $a^{k-1} x \neq a^{k-1} y$ and so $\lambda_{a}$ is not injective. 
CoROLlary 4.2. Let $S$ be right subdirectly irreducible. If $a \in S$ is aperiodic then $|a \rho(x)|=1$ for all $x \in S$.

Proof. By Lemma 2.1, if $|a \rho(x)|>1$ then $\lambda_{a}$ is injective, whence $a$ is periodic.

CoROLlary 4.2. Let $S$ be right subdirectly irreducible. Then $\lambda_{a}$ is injective iff $S$ has an identity and $a \in G$.

Proof. If $S$ has an identity then $\lambda_{a}$ is injective for all $a \in G$. On the other hand, if $\lambda_{a}$ is injective then $a$ is periodic, say $a^{n}=a^{m}$ for some $n, m \in \mathscr{N}, n \neq m$. But then $a^{n-1}=a^{m-1}$. By induction we obtain $a=a^{k}$ for some $k>1$ and so $a^{k-1} a=a$. Since $a \neq 0$, this implies that $a^{k-1}=1$ whence $S$ has an identity and $a \in G$.

LEMMA 4.3. Let $M$ be a right subdirectly irreducible monoid. If $K^{2}=K$, then $a K=K$ for all nonzero $a \in I$.

Proof. If $M$ has no zero the result is obvious. Suppose then that $0 \in M$. If $a K \neq K$ for some $a \in I$, then $a K=0$. Thus the right ideal $\{x \in S \mid x K=0\}$ is nontrivial and so contains $K$, whence $K^{2}=0$.

CoROllary 4.4. Let $M$ be a right subdirectly irreducible monoid. If $K^{2}=K$ then $I \backslash 0$ has no periodic elements.

Proof. If $a \in I$ is periodic then $0 \in\langle a\rangle$ and so $a K=0$.

CoROllaRY 4.5. Let $M$ be a commutative right subdirectly irreducible monoid with $K^{2}=K$. Then $M$ is a subgroup of the $p^{\infty}$-group.

Proof. Since $K^{2}=K$ we have $a K=K$ for all $a \in K$. Thus $K a=K$ for all $a \in K$ and so $K$ is a group. Thus $M=K$ or $K=\{0\}$. Since $|K|>1$, we have $M=K$ and so $M$ is an abelian subdirectly irreducible group. The result follows from Theorem 5.1 of [5].

The case of a right subdirectly irreducible semigroup $S$ for which $K^{2}=K, K$ not a group, is very interesting. Since $K^{2}=K$ we know that $a K=K$ for all $a \in K$. However, by Corollary 4.5 and Theorem 4.1, $\lambda_{a}$ is not injective and so by Lemma 2.1, $|a \rho(x)|=1$ for all $x \in S$. Thus each nontrivial $\rho$-class is collapsed by $\lambda_{a}$. If $S$ is a monoid with $|G|>1$, then $K \backslash 0$ is the union of nontrivial $\rho$-classes, each of size $p$. Thus there is a great deal of collapsing by $\lambda_{a}$, yet 
$a K=K$

LEMMA 4.6. Let $M$ be a periodic right subdirectly irreducible monoid which is not a group. Then $K$ is the annihilator of $I$ and $K=a G \cup 0$ for any $a \in K \backslash 0$.

Proof. For each $a \in I \backslash 0,\{x \mid a x=0\}$ is a nontrivial right ideal and so contains $K$. Thus $a K=0$ for all $a \in I$, i.e., $I K=0$. Now let $a \in K \backslash 0$ whence $a I \subset K$. If $a I \neq 0$ then $a I=K$ whence $a t=a$ for some $t \in I$. But then $a t^{n}=a$ for all $n \in \mathscr{N}$ and so $a=0$, a contradiction. Thus $a I=0$ for all $a \in K$, whence $K I=0$.

By Lemma 3.10, $K=a G \cup 0$ for each $a \in K \backslash 0$. Let $A=\{x \in$ $M \mid x I=I x=0\}$. Then $K \subset A \subset I$. If $b \in A \backslash 0$ then $b G \cup 0$ is a nontrivial right ideal. But then $a G \cup 0 \subset b G \cup 0$ and so $a=b g$ for some $g \in G$, whence $b=a g^{-1} \in K$. Thus $A=K$.

If $M$ is a right subdirectly irreducible monoid for which $G$ is finite and $|G|>1$, then $G$ is a cyclic group of prime power, or $G$ is a generalized quaternion group. Moreover, if $M$ is periodic then since $G$ acts semiregularly on $K \backslash 0$, (if $M \neq G$ ), we have $|K|=1+|G|$.

THEOREM 4.7. If $M$ is a finite right subdirectly irreducible monoid which is not a group, then $|M| \equiv 1 \bmod (|G|)$.

Proof. $G$ acts semiregularly on $M \backslash 0$ by Lemma 3.2.

LEMMA 4.8. If $M$ is a finite right subdirectly irreducible monoid which is not a group, then $I^{n}=K$ for some $n \in \mathscr{N}$. For all $x \in I^{n-1} \backslash K, x I=K$.

Proof. Let $n$ be such that $I^{n} \neq 0$ but $I^{n+1}=0$. Then $K \subset I^{n}$. For $x \in I^{n}$ we have $x I=I x=0$ and so $x \in K$. Thus $K=I^{n}$. If $x \in I^{n-1} \backslash K$, then $x I \neq 0$ by Lemma 3.11 and so $K \subset x I \subset I^{n}=K$. Thus $x I=K$.

Thus if $M$ is a finite right subdirectly irreducible monoid which is not a group, then there exists $n \in \mathscr{N}$ and that

$$
I \supsetneqq I^{2} \supsetneqq \cdots \supsetneqq I^{n-1} \supsetneq I^{n}=K \text {. }
$$

LEMMA 4.9. If $M$ is a finite right subdirectly irreducible monoid which is not a group, then $\left|I^{i}\right| \equiv 1(\bmod |G|)$ for all $i \in \mathscr{N}$.

Proof. $G$ acts semiregularly on $I^{i} \backslash 0$. 
Corollary 4.10. For each $i,\left|I^{i} \backslash I^{i+1}\right| \equiv 0(\bmod |G|)$.

Given any $m, n \in \mathscr{N}, n>1$ there exists a right subdirectly irreducible monoid $M$ which is not a group, and for which $|M|=$ $1+n p^{m}$.

ExAmPLe 4.11. Let $m \in \mathscr{N}$ and let $G$ be a group of order $p^{m}$ with minimum subgroup $H \neq 1$. Let $n \in \mathscr{N}$ and define $G_{0}=G_{1}=\cdots=$ $G_{n}=G$. Define $M$ to be the disjoint union $S=G_{0} \cup G_{1} \cup \cdots \cup G_{n} \cup 0$. Multiplication in $M$ is defined as follows:

if $(g)_{i} \in G_{i},(h)_{j} \in G_{j}$ then $(g)_{i}(h)_{j}=\left\{\begin{array}{cc}(g h)_{i+j} & i+j \leqq n \\ 0 & i+j>n .\end{array}\right.$

Then $M$ is a right subdirectly irreducible monoid with $G=G_{0}$, $I=G_{1} \cup G_{2} \cup \cdots \cup G_{n} \cup 0, K=G_{n} \cup 0, I^{n}=K$, and $|M|=1+(n+1) p^{n}$. Note that $\left|I^{i} \backslash I^{i+1}\right|=|G|$ for $1 \leqq i \leqq n$. It is clear that the example can be modified in such a manner that $\left|I^{i} \backslash I^{i+1}\right|=n_{i}|G|$ for $1 \leqq i \leqq n-1, n_{i}$ arbitrary.

\section{REFERENCES}

1. G. Baird and G. Thierrin, On semigroups in which every Rees one-sided congruence is a congruence, Semigroup Forum, 4 (1972), 215-223.

2. W. H. Carlisle, Subdirectly irreducible H-semigroups, Semigroup Forum, 9 (1974), 28-35.

3. D. B. McAlister and L. O'Carroll, Maximal homomorphic images of commutative semigroups, Glasgow Math. J., 12 (1971), 12-17.

4. S. A. Rankin, C. M. Reis and G. Thierrin, Right local semigroups, to appear in the J. of Algebra.

5. B. M. Schein, Homomorphisms and subdirect decompositions of semigroups, Pacific J. Math., 17 (1966), 529-547.

Received June 14, 1976. Research for the first and third authors has been supported by Grants A8218 and A7877 to the respective authors by the National Research Council of Canada.

The UNIVERsity OF WeSTERN ONTARIO

LONDON, CANADA N6A 5B9 


\section{PACIFIC JOURNAL OF MATHEMATICS}

\section{EDITORS}

DONALD BABBITT (Managing Editor)

University of California

Los Angeles, CA 90024

HUgo RossI

University of Utah

Salt Lake City, UT 84112

C. C. Moore and ANDrew OGG

University of California

Berkeley, CA 94720

\section{J. DUGUNDJI}

Department of Mathematics

University of Southern California

Los Angeles, CA 90007

R. FinN and J. MILGRaM

Stanford University

Stanford, CA 94305

\section{ASSOCIATE EDITORS}
E. F. BECKENBACH
B. H. NeumanN
F. WOLF
K. YOSHIDA

\section{SUPPORTING INSTITUTIONS}

UNIVERSITY OF BRITISH COLUMBIA CALIFORNIA INSTITUTE OF TECHNOLOGY

UNIVERSITY OF CALIFORNIA

MONTANA STATE UNIVERSITY

UNIVERSITY OF NEVADA, RENO

NEW MEXICO STATE UNIVERSITY

OREGON STATE UNIVERSITY

UNIVERSITY OF OREGON
UNIVERSITY OF SOUTHERN CALIFORNIA

STANFORD UNIVERSITY

UNIVERSITY OF HAWAII

UNIVERSITY OF TOKYO

UNIVERSITY OF UTAH

WASHINGTON STATE UNIVERSITY

UNIVERSITY OF WASHINGTON

The Supporting Institutions listed above contribute to the cost of publication of this Journal, but they are not owners or publishers and have no responsibility for its content or policies.

Mathematical papers intended for publication in the Pacific Journal of Mathematics should be in typed form or offset-reproduced, (not dittoed), double spaced with large margins. Please do not use built up fractions in the text of the manuscript. However, you may use them in the displayed equations. Underline Greek letters in red, German in green, and script in blue. The first paragraph or two must be capable of being used separately as a synopsis of the entire paper. Please propose a heading for the odd numbered pages of less than 35 characters. Manuscripts, in triplicate, may be sent to any one of the editors. Please classify according to the scheme of Math. Reviews, Index to Vol. 39. Supply name and address of author to whom proofs should be sent. All other communications should be addressed to the managing editor, or Elaine Barth, University of California, Los Angeles, California, 90024.

50 reprints to each author are provided free for each article, only if page charges have been substantially paid. Additional copies may be obtained at cost in multiples of 50 .

The Pacific Journal of Mathematics is issued monthly as of January 1966. Regular subscription rate: $\$ 84.00$ a year (6 Vols., 12 issues). Special rate: $\$ 42.00$ a year to individual members of supporting institutions.

Subscriptions, orders for numbers issued in the last three calendar years, and changes of address should be sent to Pacific Journal of Mathematics, P.O. Box 969, Carmel Valley, CA 93924, U.S.A. Older back numbers obtainable from Kraus Periodicals Co., Route 100, Millwood, NY 10546.

PUBLISHED BY PACIFIC JOURNAL OF MATHEMATICS, A NON-PROFIT CORPORATION

Printed at Kokusai Bunken Insatsusha (International Academic Printing Co., Ltd.). 8-8, 3-chome, Takadanobaba, Shinjuku-ku, Tokyo 160, Japan.

Copyright (C) 1979 by Pacific Journal of Mathematics Manufactured and first issued in Japan 


\section{Pacific Journal of Mathematics}

\section{Vol. 85, No. $2 \quad$ October, 1979}

Charles A. Akemann and Steve Wright, Compact and weakly compact derivations of $C^{*}$-algebras ........................... 253

Dwight Richard Bean, Andrzej Ehrenfeucht and George Frank McNulty, Avoidable patterns in strings of symbols ................... 261

Richard Clark Brown, Notes on generalized boundary value problems in Banach spaces. I. Adjoint and extension theory.................

Kenneth Alexander Brown and John William Lawrence, Injective hulls of group rings .................................... 323

Jacob Burbea, The Schwarzian derivative and the Poincaré metric ....... 345

Stefan Andrus Burr, On the completeness of sequences of perturbed polynomial values ....................................

Peter H. Chang, On the characterizations of the breakdown points of quasilinear wave equations..............................

Joseph Nicholas Fadyn, The projectivity of $\operatorname{Ext}(T, A)$ as a module over

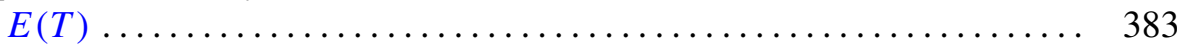

Donald Eugene Maurer, Arithmetic properties of the idèle discriminant .... 393

Stuart Rankin, Clive Reis and Gabriel Thierrin, Right subdirectly irreducible semigroups ................................. 403

David Lee Rector, Homotopy theory of rigid profinite spaces. I ........ 413 Raymond Moos Redheffer and Wolfgang V. Walter, Comparison theorems for parabolic functional inequalities................

H. M. (Hari Mohan) Srivastava, Some generalizations of Carlitz's theorem

James Alan Wood, Unbounded multipliers on commutative Banach algebras....

T. Yoshimoto, Vector-valued ergodic theorems for operators satisfying norm

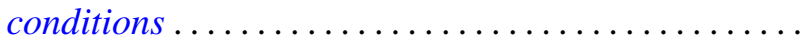

Jerry Searcy and B. Andreas Troesch, Correction to: "A cyclic inequality and a related eigenvalue problem".

Leslie Wilson, Corrections to: "Nonopenness of the set of Thom-Boardman maps" 